preface, Prof. Abhyankar states that his interest in analytic geometry was aroused by his researehes into the extension of this result to complete intersections, rather than just hypersurfaces. This extension was achieved by making use of Weiorstrass' preparation theorem. After publishing this, he became convinced that the entire local theory of complex spaces could be treated quite simply by first invoking Weierstrass' preparation theorem and then using Krull's technique of local rings together with some methods from algebraic geometry us developed by o. Zariski. This volume is the result.

There is happily no Chapter 0 . The first chapter contains the elementary theory of the complex space $C^{n}$ and includes sections on Laurent expansions and Cauchy theory for functions of several variables. Chapter 2 covers Weierstrass' preparation theorem, unique factorization in power series rings, the implicit function theorem, Hensel's lemma, the Riemann extension theorem, and results on the connectivity of algebroid hypersurfaces. Chapter 3 is a review of local algebra, and includes important results concerning Henselian rings.

The next two chapters form the central part of the book. Chapter 4 develops analytic geometry at a point (parameters for ideals, regularity of quotient rings, etc.) and this is extended locally (that is, in the neighbourhood of a point) in Chapter 5. The latter deals with a number of interesting topies, including the pure-dimensionality of an analytic set in a neighbourhood of a point at which it is irreducible, the parametrization of an irreducible analytic set germ, normal points and singular loci, a few useful comments on algebraic varieties and some connectivity properties of complex analytic sets.

Chapter 6 is concerned with the language of sheaves and is one of the first reasonably adequate treatments of this subject in the English language. The subject is pursued as far as the theory of $A$-modules (=sheaves of modules over a sheaf of rings $A$ ) which are locally finitely presented, and covers the variations on the notion of coherence. Serre's basic theorem, on the coherence properties of a short exact sequence of $A$-modules, is proved in full. Using the sheaf language, the final chapter goes on to recapitulate the previous theory in the 'global' context of analytic spaces and culminates in an account of bimeromorphic maps and normalizations.

The book can scarcely be recommended to a tyro in this subject. It is "full of mathematics", indigestibly so at times, and little attempt has been made to give the reader any motivation for what is happening. (The indigestible style reaches a peak of unintelligibility in the last paragraph of p. 376.) Nevertheless, the book must be welcomed as a significant addition to the literature on its subject. It is well printed, well indexed, and contains a good bibliography. A projected second volume on Stein spaces and the global aspects of complex spaces should prove a valuable complement. $\quad$ J. A. TyrrelL

\section{NOBEL LECTURES IN PHYSIOLOGY OR MEDICINE}

Nobel Lectures in Physiology or Medicine, 1942-1962

Pp. xiv +839 . (Amsterdam, London and New York: Elsevier Publishing Company, 1964.) $160 s$.

$\mathrm{T}$

HE publication in English of the collected Nobel Prize lectures in volumes by subjects will make these available to a much wider circle than the earlier publication in the language in which they were given in annual volumes.

The title gives the scope of the present volume: in it are 36 lectures, some 40 biographies of the prize winners, and 20 presentation addresses giving the background of the work of each year's award. Many of the lectures are fully illustrated with diagrams and plates.
The Nobel Prizes for 1942-62 were given for work done during the thirties and up to the 'sixties. This has boen a period of most rapid advance in both physiology and medicine; the Nobel lectures not unnaturally give a cross-section of many growing-points in this very active field. The lectures are not only of great scientific interest but also make extremely interesting reading for the nonspecialist, as their presentation is less technical than the same authors would have used in their scientific papers, and a formidable mass of new knowledge is brought into an easily assimilable form.

Modern medical advance depends on the activity of the sciences fundamental to medicine; only after a discovery has been established beyond doubt in the laboratory can it be tested in a clinical setting. Thus, entomology and chemistry led to DDT, for which Müller received the Prize in 1948, and as with many discoveries in the present lectures this was rapidly applied throughout the world and revolutionized man's fight against insect-borne diseases. In the Second World War it became an important weapon possessed by the Allies alone and undoubtedly greatly reduced loss of life in later campaigns of the War. Others, such as the work of Gasser and Erlanger (1944), are less spectacular but no less important. Their work on nervous conduction is a striking example of the effect of fundamental knowledge in fructifying related fields. The important role of fine nerve fibres has been uncovered in much recent work, and is not, as was earlier believed, only to convey impulses subserving pain and temperature. The resulting fuller understanding of fibre size and function has already become part of the background of clinical neurology and neurophysiology.

It is difficult to pick topics for special mention in a short review. A striking discovery which has been applied rapidly throughout the world was the isolation of penicillin and the development of similar bactericidal agents which have become available following Fleming, Chain and Florey's discoveries. Thus, the Nobel Prize to the authors of penicillin was followed in 1952 by that to Selman Waksman for his work on the isolation of streptomycin. Behind such rapidly applicable discoveries are the fundamental biochemical discoveries such as those of Krebs and Lipmann, for which the Prize was awarded in 1953, and Theorell's work on oxidation enzymes (Prize, 1955). In the more recent lectures the structure of nucleic acids, genetic codes, and genos and chemical reactions are prominent, with the Prize in 1958 to Beadle, Tatum and Lederberg; Ochoa and Kornberg (1959); Crick, Watson and Wilkins in the last award with which this volume deals (1962).

Some of the lectures provide lively reading apart from their scientific content, such as that by Bekesy (1961), in which he gives a charming exposition of how he came to unravel the mechanies of the cochlea.

One general feature stands out from these collected works and that is to how great an extent medical advance is now determined in laboratories and by animal experiments, from which it must penetrate slowly to the wards. Of the 35 contributions, few deal with matters into which patients enter, but no one can doubt that many of these fundamental discoveries are already influencing practical medicine, and that others will have a very great influence in the years to come.

The book is expensive (160s.), but it is to be hoped that it will be available to many via libraries, as the inspiration through reading of discoveries in the discoverers' own words, brings life to many subjects that a student needs to master. Many of the lectures are models of scientific exposition. The general interest will assure it a place on many scientists' shelves. All concerned are to be congratulated on this noble work. The subject matter is not unnaturally remarkable, but the pub. lishers have done their part in a way worthy of its outstanding content.

Bryan H. C. Matthews 